\title{
High Resolution 802.11ax-based Passive Radar for Human Movement Monitoring
}

\author{
Hasan Can Yildirim ${ }^{\# 1}$, Laurent Storrer ${ }^{\# 2}$, François Rottenberg*\#3 \\ Jerôme Louveaux ${ }^{* 4}$, Philippe De Doncker ${ }^{\# 5}$, François Horlin ${ }^{\# 6}$ \\ \#Université Libre de Bruxelles, Belgium \\ *Université Catholique de Louvain, Belgium \\ $\left\{{ }^{1}\right.$ hayildir, ${ }^{2}$ lstorrer, ${ }^{5}$ pdedonck, ${ }^{6}$ fhorlin $\} @$ ulb.ac.be, $\left\{{ }^{3}\right.$ francois.rottenberg, ${ }^{4}$ jerome.louveaux $\} @$ uclouvain.be
}

\begin{abstract}
Passive Radars, based on the emerging 802.11ax Wi-Fi standard, are considered for indoor human movement detection. Since the Wi-Fi access points transmit multiple frames in bursts, the FFT-based Doppler estimation techniques fail due to the limited duration of the bursts. Therefore, super resolution techniques are examined for low Doppler frequency estimation based on a small number of frames. An algorithm is proposed which uses ESPRIT in an iterative fashion. The performance of the algorithm is numerically analysed, compared to theoretical bounds, and validated experimentally.
\end{abstract}

Keywords - Passive Radar, 802.11ax, Wi-Fi sensing, Doppler frequency estimation, indoor human detection

\section{INTRODUCTION}

Passive radars (PR) are devices that make use of the existing communication signals to detect and track targets in the environment. In the classical radar processing, the signal bandwidth and duration determine the range and speed resolution, respectively. Wi-Fi 6 (802.11ax) [1] is a relevant signal of opportunity (SO) for PRs, due to the availability of wide bandwidths up-to $160 \mathrm{MHz}$. Since the Wi-Fi signals are modulated with the Orthogonal Frequency Division Multiplexing (OFDM), the classical radar processing for range estimation can be replaced by channel impulse response (CIR) estimation to improve the range accuracy [2]. To do so, the transmitted symbols need to be known. The range processing is followed by windowing to reduce the leakage in the frequency domain, fast Fourier transform (FFT) to obtain the Doppler spectrum and the constant false alarm ratio (CFAR) to separate the target peaks from the noise.

However, Wi-Fi access points (AP) work by transmitting bursts of OFDM frames. Therefore, the duration of the SO may not be long enough to achieve the desired speed resolution. The latter problem is discussed within the recently formed Task Group for Wi-Fi Sensing [3], that aims at indoor monitoring of human movements based on the Wi-Fi signals. To estimate the human main-body speed with an average velocity of 1.4 $\mathrm{m} / \mathrm{s}$, approximately $0.5 \mathrm{~m} / \mathrm{s}$ speed resolution is required. Thus, the measured duration of the SO has to be at least $50 \mathrm{~ms}$ at 5.6 GHz for the FFT-based processing, which is an unrealistic assumption for Wi-Fi systems.

In this work, we address the limitations of the classical processing method on the accuracy of the speed estimation. Since the frequency resolution of the FFT-based method is limited by the measured duration of the SO, parametric estimators with super-resolution may constitute an interesting alternative to achieve better accuracy for the speed estimation. In [4], by exploiting the unique OFDM frame structure of the Radar-Radio (RadCom) systems, the range and Doppler are estimated with ESPRIT and Least Square (LS) algorithms, respectively. However, the considered target velocities are 80 and $100 \mathrm{~m} / \mathrm{s}$, which yield high Doppler frequencies. In [5], joint estimation of angle and Doppler frequency are performed using the ESPRIT algorithm for a bistatic multi-antenna radar. When the number of targets is increased and/or the number of antennas is decreased, the algorithm performs poorly. In [6], 802.11p signals are used for radar processing, where the delay and Doppler are estimated with ESPRIT, achieving sub $0.05 \mathrm{~m} / \mathrm{s}$ accuracy. However, the observation time is considered to be around $50 \mathrm{~ms}$, which already provides high accuracy with the FFT-based method. Moreover, it is also mentioned that, for targets with weak reflectivity (such as humans), it is difficult for ESPRIT to estimate the speeds between -2 and $2 \mathrm{~m} / \mathrm{s}$. The goal of our work is to develop a new iterative algorithm inspired by ESPRIT, which provides accurate Doppler frequency estimation to enable the indoor monitoring of human movements, where the source of the SO is considered to be an 802.11ax AP. We both numerically and experimentally demonstrate the efficiency of the algorithm.

This paper is structured as follows. In section II, the OFDM frame structure and radar processing are briefly explained. In section III, the ESPRIT algorithm is introduced and the proposed algorithm is explained in details. In section IV, the performance of the algorithms is compared numerically, and the results are validated experimentally. Finally, in section V, the conclusion is drawn.

The following notations are used in the paper: superscripts $H$ and $T, \mathbf{I}, \operatorname{diag}(\mathbf{A}), \mathbf{A}_{*, i}$ and $\bullet$ are defined as the Hermitian and matrix transpose operators, the identity matrix, the vector containing the diagonal elements of an arbitrary matrix $\mathbf{A}, i$-th column of $\mathbf{A}$ and the Hadamard product, respectively.

\section{OFDM SIGNALS AND RADAR PROCESSING}

The considered OFDM frame is composed of two fields: the preamble and the data, composed of 8 and 32 OFDM symbols, respectively. The preamble is used for the synchronization and the channel estimation to obtain the transmitted 
data symbols. In each frame, as shown in Figure 1, all 40 OFDM symbols can be used for PR processing, assuming that the data is reconstructed by the PR working as a conventional $\mathrm{Wi}-\mathrm{Fi}$ receiver for that purpose. Moreover, one OFDM frame duration $(504 \mu \mathrm{s})$ corresponds to approximately $2 \mathrm{kHz}$ Fourier resolution, yielding $53 \mathrm{~m} / \mathrm{s}$ speed resolution at $5.6 \mathrm{GHz}$. To achieve $0.5 \mathrm{~m} / \mathrm{s}$ resolution with the FFT-based method, approximately 105 frames are required.

\begin{tabular}{|c|c|c|}
\multicolumn{1}{c|}{8 Symbols } & \multicolumn{2}{c|}{32 Symbols } \\
\hline Preamble & Data & Next Frames \\
\hline $108.8 \mu \mathrm{s}$ & $435.2 \mu \mathrm{s}$ & \\
\hline \multicolumn{2}{|c|}{ One Frame }
\end{tabular}

Fig. 1. The OFDM frame structure, which is identical for the following frames.

The transmit signal corresponding to $M$ consecutively transmitted OFDM symbols composed of $Q$ subcarriers can be written as

$$
s(t)=\sum_{m=0}^{M-1} \sum_{q=0}^{Q-1} X[q, m] e^{j 2 \pi \frac{q}{T_{u}}\left(t-m T_{o}\right)} u\left(t-m T_{o}\right)
$$

where $X[q, m]$ is the complex PSK/QAM symbol mapped on the $q$-th subcarrier of the $m$-th OFDM symbol. $T_{u}=Q / B$, where $B$ is the system bandwidth, is the duration of one OFDM symbol. $T_{o}=T_{u}+T_{c p}$ is the OFDM symbol duration including the cyclic prefix (CP) of duration $T_{c p}$. The rectangular pulseshaping function, $u(t)$ is defined as

$$
u(t)= \begin{cases}1, & 0 \leq t<T_{o} \\ 0, & \text { elsewhere }\end{cases}
$$

In a quasi monostatic configuration where the transmitter and the PR are co-located, the time-varying CIR can be modelled as

$$
h(\tau, t)=\sum_{j=1}^{J} \beta_{j}^{c} \delta\left(\tau-\tau_{j}^{c}\right)+\sum_{l=1}^{L} \beta_{l} \delta\left(\tau-\tau_{l}\right) e^{-j 2 \pi f_{l} t}
$$

where $J$ and $L$ are the number of static and moving paths in the channel; $\beta_{j}^{c}$ and $\beta_{l}$ are the complex amplitudes (including the random phase shifts). Since we consider a quasi monostatic scenario, $\tau_{j}^{c}, \tau_{l}$ and $f_{l}$ correspond to the two-way propagation delay of static and moving objects, and the Doppler shift, respectively. The received signal can be written as

$$
r(t)=\sum_{j=1}^{J} \beta_{j}^{c} s\left(t-\tau_{j}^{c}\right)+\sum_{l=1}^{L} \beta_{l} s\left(t-\tau_{l}\right) e^{-j 2 \pi f_{l} t}+z(t)
$$

where $z(t)$ is the additive white Gaussian noise (AWGN) of zero-mean and variance $\sigma^{2}$. The goal of a radar receiver is to estimate the propagation delays $\tau_{l}$, and the corresponding Doppler shifts $f_{l}$. After the received signal is low pass filtered, sampled at a fixed rate, and the synchronization is performed, the PR can perform CIR estimation on each OFDM symbol. Then, each estimated CIR, with index $m$, is stacked on a matrix, also known as range/slow-time map. For a fixed range $d$ i.e., tap index, the slow-time response can be written as [7]

$$
h_{d}[m]=\sum_{j=1}^{J_{d}} \alpha_{j}^{c}+\sum_{p=1}^{L_{d}} \alpha_{p} e^{-j 2 \pi f_{p} m T_{o}}+z_{d}[m]
$$

where $m=0, \ldots, M-1 ; \alpha_{j}^{c}$ and $\alpha_{p}$ are the complex amplitudes obtained after the processing, while $J_{d}$ and $L_{d}$ are the total number of static and moving paths at a given distance, hence $J_{d}<J$ and $L_{d}<L, \forall d$ and $z_{d}[m]$ are the corresponding noise samples. The static objects can be cancelled by removing the mean of (4), as shown in [8]. Moreover, the moving objects are identified by their distances while the Doppler frequencies for a given distance are modelled as a sum of complex exponentials. The frequency resolution is determined by the Fourier bin width $1 / M T_{o}$ for conventional radar processing. Since the OFDM symbol duration, $T_{o}$, is fixed by the standard, the only parameter that can vary to improve the resolution is $M$, which is also limited by the nature of the Wi-Fi systems. Therefore, we propose to make use of the parametric methods to estimate the frequencies for relatively lower values of $M$.

\section{Subspace Methods for Frequency Estimation}

Let us write (4) in matrix form, such as

$$
\begin{gathered}
\mathbf{h}_{d}=\mathbf{A} \boldsymbol{\alpha}+\mathbf{z}_{d}, \in \mathbb{C}^{M \times 1} \\
\boldsymbol{\alpha}=\left[\alpha_{1} \ldots \alpha_{L_{d}}\right]^{T}, \in \mathbb{C}^{L_{d} \times 1} \\
\mathbf{A}=\left[\mathbf{a}\left(f_{1}\right) \ldots \mathbf{a}\left(f_{L_{d}}\right)\right], \in \mathbb{C}^{M \times L_{d}}
\end{gathered}
$$

where $\mathbf{h}_{d}=\left[h_{d}[0] \ldots h_{d}[M-1]\right]^{T}, \mathbf{z}_{d}=\left[z_{d}[0] \ldots z_{d}[M-1]\right]^{T}$, $\mathbf{a}(f):=\left[\begin{array}{llll}1 & e^{-j 2 \pi f T_{o}} & \ldots & e^{-j 2 \pi(M-1) f T_{o}}\end{array}\right]^{T}, \quad \in \mathbb{C}^{M \times 1}$ and $\boldsymbol{\alpha}$ contains the complex amplitudes per Doppler frequency. A is the Vandermonde matrix whose columns contain the complex sinusoids in (4). The covariance matrix of (5) can be written as $\mathbf{R}=\mathbb{E}\left\{\mathbf{h}_{d} \mathbf{h}_{d}^{H}\right\}=\mathbf{A} \mathbf{K} \mathbf{A}^{\mathbf{H}}+\sigma^{2} \mathbf{I}$, also known as the Carathéodory parametrization where $\boldsymbol{\alpha}=\operatorname{diag}(\mathbf{K})$. After performing the eigenvalue decomposition on $\mathbf{R}$, we obtain $\mathbf{U}_{s}$ which is the orthonormal eigenvectors associated with the $L_{d}$ largest eigenvalues, $\left\{\lambda_{s}\right\}$, of $\mathbf{R}$. Remaining orthonormal eigenvectors, $\mathbf{U}_{n}$, are associated with the $M-L_{d}$ smallest eigenvalues, $\left\{\lambda_{n}\right\}$, of $\mathbf{R}$, equal to $\sigma^{2}$. Matrices $\mathbf{U}_{s}$ and $\mathbf{U}_{n}$ are commonly referred to the basis vectors spanning the signal and noise subspaces, respectively. For further information about the subspace methods the reader is referred to [9].

\section{A. ESPRIT}

The algorithm works by dividing the matrix $\mathbf{U}_{\mathbf{s}}$, into two submatrices

$\mathbf{U}_{s_{1}}=\left[\begin{array}{ll}\mathbf{I}_{M-1 \times M-1} & \mathbf{0}_{1 \times M}\end{array}\right] \mathbf{U}_{\mathbf{s}}, \quad \mathbf{U}_{s_{2}}=\left[\begin{array}{ll}\mathbf{0}_{1 \times M} & \mathbf{I}_{M-1 \times M-1}\end{array}\right] \mathbf{U}_{\mathbf{s}}$

Furthermore, the following equality between the two submatrices can be derived: $\mathbf{U}_{s_{2}}=\mathbf{U}_{s_{1}} \boldsymbol{\Phi}$, where the eigenvalues of the matrix $\boldsymbol{\Phi}$ contain complete information about the frequencies in (4) [9]. In practice, the matrix $\boldsymbol{\Phi}$ is estimated with the total least square (TLS) method from an estimation of $\mathbf{R}$. Once 
the frequencies are estimated, the complex amplitudes can be estimated with LS, using $\tilde{\boldsymbol{\alpha}}=\left(\mathbf{A}^{H} \mathbf{A}\right)^{-1} \mathbf{A}^{H} \mathbf{h}_{d}$.

\section{B. Two-step ESPRIT}

ESPRIT requires the separation between the signal and the noise subspaces. Thus, the number of signals i.e., $L_{d}$, needs to be known a priori. Our approach is as follows. First, a sufficiently high number of columns of $\mathbf{U}$ are selected to create $\mathbf{U}_{s}^{e}$ containing all the signal basis vectors and at least one of the noise basis vectors. Then the submatrix separation, given in (8), is performed for two different step sizes such as

$$
\begin{aligned}
\mathbf{U}_{s_{1}}^{1}=\left[\begin{array}{ll}
\mathbf{I}_{M-1 \times M-1} & \mathbf{0}_{1 \times M}
\end{array}\right] \mathbf{U}_{s}^{e}, & \mathbf{U}_{s_{2}}^{1}=\left[\begin{array}{ll}
\mathbf{0}_{1 \times M} & \mathbf{I}_{M-1 \times M-1}
\end{array}\right] \mathbf{U}_{s}^{e} \\
\mathbf{U}_{s_{1}}^{2}=\left[\begin{array}{ll}
\mathbf{I}_{M-2 \times M-2} & \mathbf{0}_{2 \times M}
\end{array}\right] \mathbf{U}_{s}^{e}, & \mathbf{U}_{s_{2}}^{2}=\left[\begin{array}{ll}
\mathbf{0}_{2 \times M} & \mathbf{I}_{M-2 \times M-2}
\end{array}\right] \mathbf{U}_{s}^{e} \\
\mathbf{U}_{s_{2}}^{1}=\tilde{\boldsymbol{\Phi}}^{1} \mathbf{U}_{s_{1}}^{1}, & \mathbf{U}_{s_{2}}^{2}=\tilde{\boldsymbol{\Phi}}^{2} \mathbf{U}_{s_{1}}^{2}
\end{aligned}
$$

where the matrices $\tilde{\boldsymbol{\Phi}}^{1}$ and $\tilde{\boldsymbol{\Phi}}^{2}$ are separately estimated with the TLS method yielding to two sets of estimated frequencies. The estimated frequencies are rounded up to the second decimal, and only the common ones are selected. This method allows the joint estimation of the set of frequencies $\{\tilde{f}\}_{p=1}^{\tilde{L}_{d}}$ and the number of signals $\tilde{L}_{d}$.

\section{Iterative Doppler Frequency Estimator (iDoFEst)}

The proposed algorithm replaces the windowing, Doppler FFT and CFAR by iteratively estimating the Doppler frequencies. The only required input to the algorithm is the vector given in (5). The block diagram of the algorithm is given in Figure 2.

First, the covariance matrix of (5) and the eigenvalue decomposition are computed. The set of frequencies and the number of signals are estimated by the 2-step ESPRIT. Corresponding Vandermonde matrix, $\tilde{\mathbf{A}}$, is constructed from the estimated frequencies, as in (7), and the amplitudes, $\tilde{\boldsymbol{\alpha}}$, are estimated with the LS method through (5). Then, a confidence analysis is performed to determine the accuracy of each estimated frequency. To do so, instead of comparing the amplitudes against a threshold [9], the estimated signals are projected to the noise subspace $\mathbf{U}_{n}$ since the noise projection enlarges the estimation errors to be spotted more easily. To perform the confidence analysis, the constructed signals in $\tilde{\mathbf{A}}$ are scaled by $\tilde{\boldsymbol{\alpha}}$, and the result is projected onto the noise subspace, yielding

$$
\mathbf{C}=(\tilde{\mathbf{A}} \bullet \tilde{\boldsymbol{\alpha}})^{H} \mathbf{U}_{n} \mathbf{U}_{n}^{H}, \in \mathbb{C}^{\tilde{L}_{d} \times M}
$$

and the rows of the matrix $\mathbf{C}$ are averaged to obtain the vector $\mathbf{c} \in \mathbb{C}^{\tilde{L}_{d} \times 1}$ whose elements, $c_{i}$, contain the confidence per estimated frequency. Then, each $c_{i}$ is compared with a numerically obtained threshold. If any given $c_{i}$ is higher than the threshold, the corresponding estimated frequency $f_{i}$, amplitude $\boldsymbol{\alpha}_{i}$ and the constructed sinusoid $\tilde{\mathbf{A}}_{*, i}$, i.e. $\boldsymbol{a}\left(f_{i}\right)$, are discarded. Otherwise, the estimations are kept as accurate. After the confidence analysis, if the number of columns of $\tilde{\mathbf{A}}$ has not changed, all the estimations have high accuracy, therefore the algorithm converges. Otherwise, the remains of the matrix $\tilde{\mathbf{A}}$ and the vector $\tilde{\boldsymbol{\alpha}}$ are multiplied, yielding to the vector $\tilde{\mathbf{h}}^{\prime}$, which corresponds to the sum of all the accurately estimated signals. Finally, this vector is subtracted from the input vector yielding the residual $\tilde{\mathbf{h}}^{r}$. The algorithm is repeated with the residual until convergence. In order to guarantee the convergence, the number of iterations can be fixed.

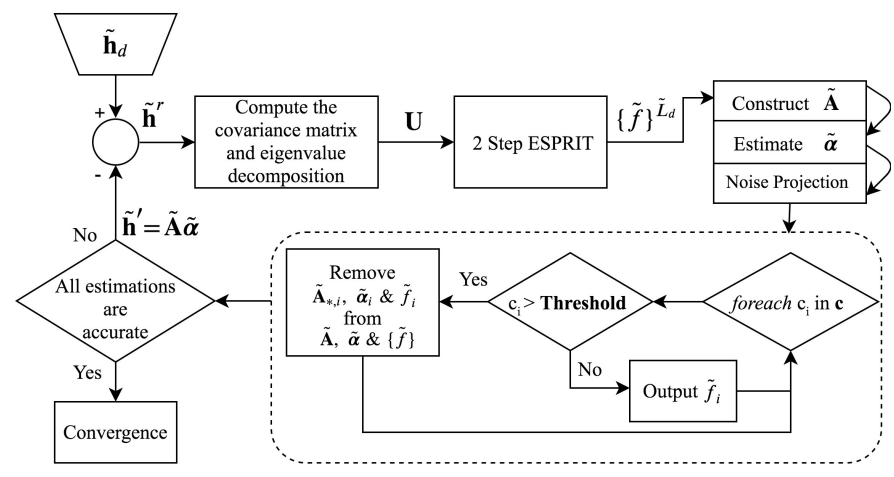

Fig. 2. Iterative Doppler Frequency Estimator

\section{NUMERICAL AND EXPERIMENTAL RESULTS}

In this section, we numerically investigate the performance of the proposed algorithm for different scenarios. Then, the performance of the algorithm is validated by an experimental setup. The parameters, used in both numerical analyses and experimental setup, are selected based on the 802.11ax standard. The system bandwidth is set to $100 \mathrm{MHz}$. The number of subcarriers $(Q)$ and $\mathrm{CP}$ length are 1024 and 64, respectively. Carrier Frequency $\left(F_{c}\right)$ is $5.6 \mathrm{GHz}$ while each Wi-Fi frame consists of 40 OFDM symbols. The transmit power is $20 \mathrm{dBm}$ while the LNA and amplifier gain at the receiver are $23 \mathrm{~dB}$ and $20 \mathrm{~dB}$, respectively. Finally, the antenna gains are $2 \mathrm{dBi}$.

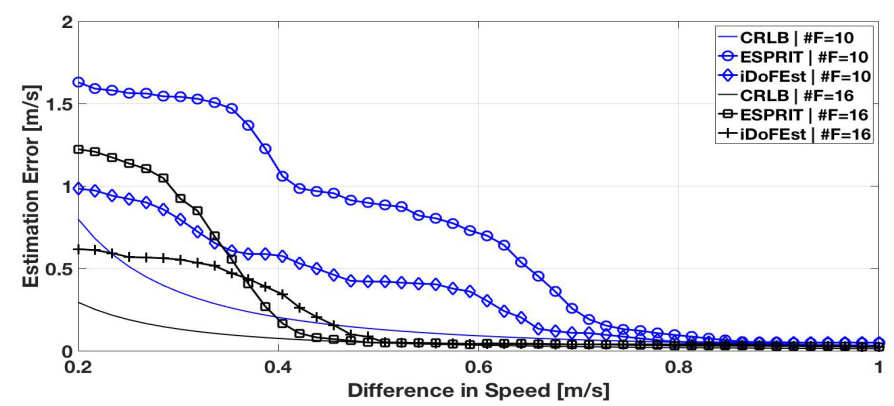

Fig. 3. Performance comparison of the algorithms for different relative differences in speed and frame sizes \#F.

In Figure 3, we consider a scenario composed of 2 targets in the environment, both modelled as single moving points. First target is moving at $0.6 \mathrm{~m} / \mathrm{s}$, while the speed of the second target varies between 0.8 and $1.6 \mathrm{~m} / \mathrm{s}$. The error on the speed estimation of the second target is plotted as a function of difference in speed for two different frame sizes. The CramerRao Lower Bound (CRLB) of the model given in (4) is also plotted [10]. Regardless of the frame size, ESPRIT has low accuracy at low differences in speed. Once the relative speed 
reaches to a sufficiently high value, both algorithms converge to an error floor.

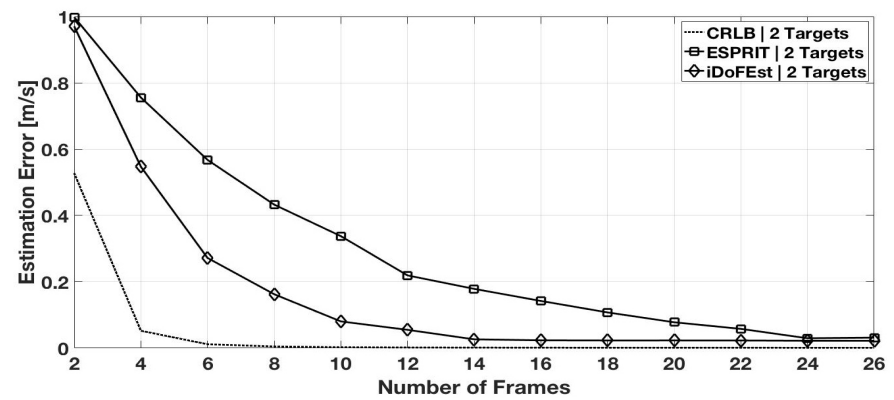

Fig. 4. Comparison of the algorithms for varying frame sizes. For each frame size, 25 different target speeds are randomly selected between 0.25 and 1.5 $\mathrm{m} / \mathrm{s}$, and 500 realizations are performed for every set of target speeds.

In Figure 4, the performance of the algorithms is compared for varying number of frames. For each realization, the target speeds are randomly selected between 0.25 and $1.5 \mathrm{~m} / \mathrm{s}$, then the average estimation error is plotted. The proposed algorithm requires lower number of frames to achieve similar performances as ESPRIT. Since the considered frequencies are low (between 10 and $55 \mathrm{~Hz}$ ), a small change in the frequency does not significantly alter the signals. Therefore, the 2step ESPRIT inaccurately estimates the number of Doppler frequencies, yielding to target misses and/or estimation errors. Meanwhile, once a set of Doppler frequencies are estimated and removed from the initial vector by the proposed algorithm, the remaining Doppler frequencies are revealed. Therefore, the algorithm shows similar performances with lower number of frames.
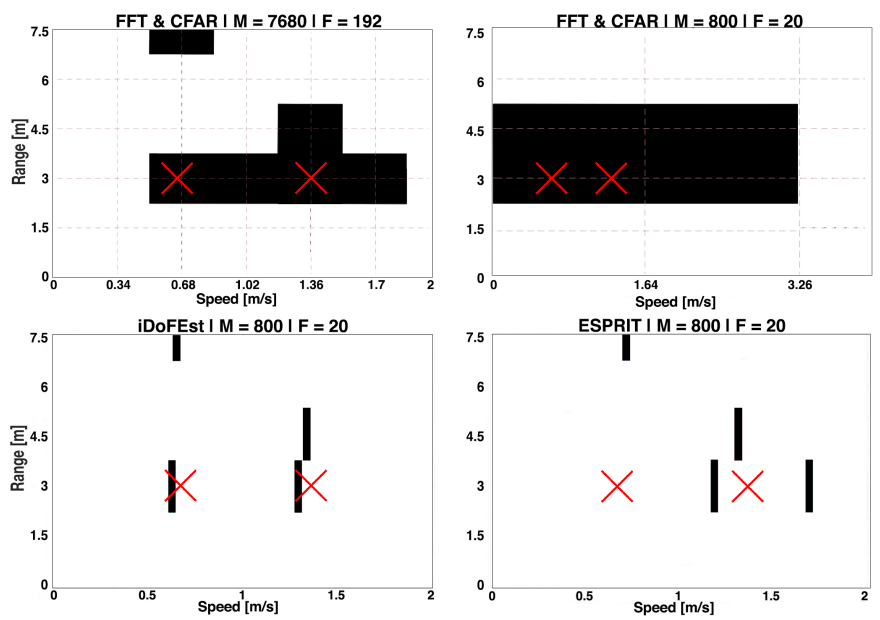

Fig. 5. Red crosses correspond to the approximate speed of the individuals, while the black areas correspond to the target detections by the corresponding algorithm. Blackman window is applied on the Doppler spectrum for the FFTbased method.

In Figure 5, the two algorithms are compared experimentally. The experiment is composed of two Software Defined Radios (SDR); one acting as the AP, the other acting as the PR. The measurements are obtained while two humans are moving towards the PR in a $4 \mathrm{~m}-\mathrm{by}-7 \mathrm{~m}$ room. The CFAR output of the FFT-based method reveals the two targets at 3 meters distance when $M$ is sufficiently high. The additional detections along the horizontal and vertical axes correspond to the artefacts due to the windowing and the multipath components of the targets, respectively. At low $M$, it is clear that FFT-based method fails to identify the targets. With the high resolution algorithms, the number of targets are correctly estimated, thanks to the 2-step ESPRIT. When there is only one target, the two algorithms show similar performances. On the other hand, the proposed algorithm out performs the 2-step ESPRIT when the number of targets in a given distance is more than one. Even though numerical analysis plotted in Figure 3, shows that 16 frames is enough to have accurate estimations when the relative difference in speed is $0.6 \mathrm{~m} / \mathrm{s}$, the proposed algorithm requires at least 20 frames to work. The main reason is the extra Doppler frequencies caused by the motion of the limbs, additional to Doppler frequency caused by the main body movement.

\section{CONCLUSiON}

In this work, high resolution algorithms are examined to estimate the Doppler frequency of the human main-body motion with a PR based on Wi-Fi signals for indoor monitoring. To achieve the required estimation accuracy, a new algorithm is proposed which is based on the high resolution ESPRIT algorithm. We numerically showed that the proposed algorithm can be used for low Doppler frequency estimation when the available number of OFDM frames is low. The performance of the algorithm is validated with the experimental setup when there are two individuals in the environment, and it is proven to be useful for indoor monitoring of the human motion.

\section{REFERENCES}

[1] Aruba Networks White Paper https://www.arubanetworks.com/assets/ wp/WP_802.11AX.pdf , Last access: 21/12/19

[2] H. C. Yildirim, L. Storrer, M. Van Eeckhaute, C. Desset, J. Louveaux and F. Horlin, "Passive Radar based on 802.11ac Signals for Indoor Object Detection", IEEE EURAD 2019.

[3] IEEE 802.11, "Wi-Fi Sensing", https://mentor.ieee.org/802.11/ documents?is_dcn=DCN\%2C\%20Title $\% 2 \mathrm{C} \% 20$ Author $\% 20 \mathrm{or} \%$ 20Affiliation\&is_group=SENS, Last access: 27/12/2019

[4] Jian-Feng Gu, J. Moghaddasi, and Ke Wu, "Delay and Doppler Shift Estimation for OFDM-Based Radar-Radio (RadCom) System", IEEE IWS 2015

[5] C. Yunhe, "Joint estimation of angle and Doppler frequency for bistatic MIMO radar", Electronics Letter (Vol: 46, Issue: 2, 21/01/2010)

[6] D. H. N. Nguyen and R. W. Heath Jr, "Dealy and Doppler Processing for multi-target Detection with IEEE 802.11 OFDM Signaling", IEEE ICASSP 2017

[7] H. C. Yildirim, J. Louveaux, P. De Doncker and F. Horlin, "Impact of Interference on OFDM based Radars", IEEE 91st Vehicular Technology Conference, May 2020

[8] L. Storrer, H. C. Yildirim, C. Desset, M. Bauduin, A. Bourdoux and F. Horlin, "Clutter removal for Wi-Fi-based passive bistatic radar", IEEE VTC2020-Spring, May 2020

[9] P. Stoica and R. L. Moses, "Spectral Analysis of Signals", Pearson Prentice Hall, 2005

[10] F. Rottenberg, J. Zhang and A. F. Molisch, "Channel Extrapolation in FDD Massive MIMO: Theoretical Analysis and Numerical Validation", IEEE Globecom, January 2019 